\title{
FEMSECT: an Inverse Section Model Based on the Finite Element Method
}

\author{
M. Losch, D. Sidorenko, and A. Beszczynska-Möller \\ Alfred-Wegener-Institut für Polar- und Meeresforschung, Bremerhaven, Germany
}

\begin{abstract}
A new inverse model is presented for the analysis of hydrographic section data in conjunction with velocity measurements. The model offers advantages over commonly applied interpolation techniques because it combines data and physical assumptions such as geostrophic balance in the framework of a finite element discretization. Specifically a quadratic objective function of model-data misfits is minimized to give estimates of transports together with formal error estimates. The finite element method allows the accurate representation of highly irregular bottom topography and ensures consistent interpolation of model variables to measurement points. The model is called FEMSECT for Finite Element Method SECTion model. FEMSECT also gives improved flexibility and performance over standard box models by allowing dynamic adjustment of the model variables temperature and salinity. Idealized test cases illustrate that the finite element methods solve the thermal wind equations far more accurately than standard finite difference methods, especially in the presence of steep topography. For a more realistic test, FEMSECT is applied to hydrographic CTD-section data and moored-instrument current meter measurements from an array in the Fram Strait. Transport estimates by FEMSECT prove to be more robust and less sensitive to the spatial data resolution than estimates by a conventional interpolation method that only uses information from moored instruments. FEMSECT is available as a highly portable Matlab code and can be run on an ordinary desktop computer.
\end{abstract}

\section{Introduction}

The classical analysis of hydrographic section data relies on the geostrophic balance of pressure gradient force and Coriolis force. This balance is often expressed in terms of geopotential height anomaly or the thermal wind equations. The latter relates the local horizontal density gradient on a constant height surface to the vertical gradient or shear of the velocity normal to the plane of the density gradient. Given measurements of density (as a function of measured temperature, salinity, and pressure or depth), an estimate of the geostrophic velocities can be obtained by vertically integrating the thermal wind equations, relative to a so-called reference velocity. As an educated guess, one can imagine a "level-of-no-motion" at the ocean floor or between water masses that move in opposite directions; at this level the horizontal velocity can be assumed to be small. However, without additional dynamical constraints, this reference velocity is (mathematically) completely arbitrary. Therefore, additional data or additional assumptions about the absolute velocity are necessary.

Inverse techniques have widely been used to estimate mass and property fluxes through hydrographic sections in so-called inverse box-models [e.g., Wunsch, 1978; Rintoul, 1991; Macdonald and Wunsch, 1996; Ganachaud and Wunsch, 2000]. Other inverse section models treat not only reference velocity but also temperature and salinity as independent variables [Nechaev and Yaremchuk, 1995; Yaremchuk et al., 1998, 2001; Losch et al., 2002]. Essentially, these techniques require data and corresponding model values to agree within prescribed prior error bars. This is very often achieved by a least-square fit, minimizing an objective function that is the sum of the squared differences of model and data values, weighted by appropriate covariance matrices [Wunsch, 1996]. Part of the information required in addition to the hydrographic data can be inferred from mass or property conservation principles, for example, within boxes that the hydrographic sections form, but in most cases a null space remains and further information is needed.

Copyright 2005 by the American Geophysical Union. 0148-0227/05/\$9.00
In recent years, measurements of absolute velocities have become more frequent and more reliable. Ship-mounted acoustic Doppler current profiler (ADCP) measurements can reach accuracies of $1 \mathrm{~cm} \mathrm{~s}^{-1}$ [Cisewski et al., 2003] due to improvements in the global positioning systems (GPS). There have been dedicated efforts to monitor the current system in special regions such as the Fram Strait with long-term current meter moorings [Fahrbach et al., 2001; Schauer et al., 2004]. Time series of temperature and velocity from moored instruments provide estimates of heat and volume fluxes with excellent temporal resolution. However, the main source of error in these transport estimates stems from under-resolving the spatial structure of the flow. At this point a consistent approach is necessary that combines the valuable current measurements that are still comparatively sparse in space with hydrographic data that have a good spatial coverage, but are not of high temporal resolution.

Least-square methods are a natural choice, because all available information can be combined into a synthesis: The influence of each piece of information can be controlled by carefully assigning a prior error estimate, and all derived results, for example, mass transport through a hydrographic section, are not only the best estimate in an optimal sense, but also it is straightforward to compute error estimates for these results.

Recently, finite element methods (FEM) have received considerable attention in oceanography, because the triangular discretization that is typical to FEM allows an appropriate resolution of very irregular domains at comparatively low cost. Furthermore, the variables in FEM are not only defined at discrete grid nodes, but continuously over the whole domain, because the interpolation between grid nodes is implicitly defined by so-called basis functions. Applications in oceanography include regional studies [Schlichtholz and Houssais, 1999; Dobrindt and Schröter, 2003; Myers and Weaver, 1995; Myers et al., 2004], tidal models, both regional [Walters, 1987] and global [Le Provost et al., 1998], and recently even general circulation models [Danilov et al., 2004].

The flexible discretization with triangles in the finite element method is attractive for a hydrographic section because the triangles naturally take care of the so-called bottom wedges, where conventional finite difference methods are ambiguous [e.g., Wunsch, 1996; Ganachaud,2003]. The second advantage stems from the implicitly 
defined interpolation rules that allow model variables to be mapped naturally to the data locations, thus making the modeling system highly consistent. These advantages are bought with slightly higher conceptional and computational efforts.

Here we will demonstrate how a new inverse section modelthat we call FEMSECT because it is based on the finite element method-can help to interpret current measurements in conjunction with hydrographic data. The model concept is described in Section 2; in Section 3 we discuss the discretization associated with the finite element method. Section 4 demonstrates in a realistic application in Fram Strait the capabilities of the model and conclusions are drawn in Section 5.

\section{Geostrophic Inverse Model}

\subsection{Thermal wind equations}

The inverse model calculates density from the non-linear equation of state of sea-water [Fofonoff and Millard, 1983] as a function of salinity $S$, in situ temperature $T$, and pressure $p=-g \rho_{0} z$ (or depth). $g$ is the acceleration resulting from gravity, $\rho_{0}$ a mean density, and $z$ the vertical coordinate. According to the thermal wind equation, the vertical shear of horizontal velocity is proportional to the horizontal density gradient. Integrating the thermal wind equation yields

$$
v=v_{\mathrm{ref}}-\frac{g}{\rho_{0} f}\left(\int_{-h}^{z} \frac{\partial \rho}{\partial x}(T, S, p) d z\right)
$$

with the unknown reference velocities $v_{\text {ref. }} f=2 \Omega \sin \phi$ is the Coriolis parameter that depends on latitude $\phi$. The local coordinate system is oriented along the section, so that the $x$ direction is parallel to the section and the velocity $v$ is normal to it.

\subsection{Least-squares method: Optimization and error estimation}

In order to fit the geostrophic velocity shear to the data, we define the following objective function:

$$
\begin{aligned}
\mathcal{J}= & \frac{1}{2}\left(\mathbf{T}^{*}-\Phi_{T} \mathbf{T}\right)^{T} \mathbf{W}_{T}\left(\mathbf{T}^{*}-\Phi_{T} \mathbf{T}\right) \\
& +\frac{1}{2}\left(\mathbf{S}^{*}-\Phi_{S} \mathbf{S}\right)^{T} \mathbf{W}_{S}\left(\mathbf{S}^{*}-\Phi_{S} \mathbf{S}\right) \\
& +\left(\mathbf{T}^{*}-\Phi_{T} \mathbf{T}\right)^{T} \mathbf{W}_{T S}\left(\mathbf{S}^{*}-\Phi_{S} \mathbf{S}\right) \\
& +\frac{1}{2}\left(\mathbf{v}^{*}-\Phi_{v} \mathbf{v}\right)^{T} \mathbf{W}_{v}\left(\mathbf{v}^{*}-\Phi_{v} \mathbf{v}\right) \\
& +\mathcal{R}
\end{aligned}
$$

Here, in a compact matrix notation, the variables $T, S$, and $v$ are arranged in column vectors $\mathbf{T}=\left(T_{1}, \ldots, T_{i}, \ldots\right)^{T}$, etc.. All starred variables denote data vectors, for example $T_{i}^{*}$ is the temperature at the position $\left(x_{i}, z_{i}\right)$ in the section plane. $\Phi_{\alpha}$ are linear interpolation operators (matrices) that map the model variables $\alpha=T, S, v$ to the corresponding data location. If the model grid nodes are identical to the data locations then $\Phi_{\alpha}$ is simply the unit matrix. The third term in(2), which is actually the sum of two symmetric covariance terms, allows for correlations between temperature and salinity.

The data-model misfit is weighted by weighting matrices $W_{\alpha}$ that are the inverses of prior error covariances. For a specific case, these error covariances are described in Section 4. "Regularization" $\mathcal{R}$ provides prior information where the data is not sufficient to determine a unique solution; choosing a proper regularization also speeds up the convergence of the optimization process [Thacker, 1989]. We choose to include a prior guess for the unknown reference velocities and penalize roughness of the solution by requiring the horizontal gradients of temperature, salinity, and velocity to be small. After discretization we write, again in matrix notation:

$$
\begin{aligned}
\mathcal{R}= & \frac{1}{2}\left(\mathbf{v}_{\text {ref }}-\mathbf{v}_{\text {ref }, 0}\right)^{T} \mathbf{W}_{v_{\text {ref }}}\left(\mathbf{v}_{\text {ref }}-\mathbf{v}_{\text {ref }, 0}\right) \\
& +\frac{1}{2}\left(\frac{\partial \mathbf{v}}{\partial x}\right)^{T} \mathbf{W}_{r}^{(v)}\left(\frac{\partial \mathbf{v}}{\partial x}\right) \\
& +\frac{1}{2}\left(\frac{\partial \mathbf{T}}{\partial x}\right)^{T} \mathbf{W}_{r}^{(T)}\left(\frac{\partial \mathbf{T}}{\partial x}\right) \\
& +\frac{1}{2}\left(\frac{\partial \mathbf{S}}{\partial x}\right)^{T} \mathbf{W}_{r}^{(S)}\left(\frac{\partial \mathbf{S}}{\partial x}\right)
\end{aligned}
$$

where $\partial \mathbf{v} / \partial x, \partial \mathbf{T} / \partial x$, and $\partial \mathbf{S} / \partial x$ represent the vectors of discretized horizontal derivatives of the vectors $\mathbf{v}, \mathbf{T}$, and $\mathbf{S}$. $\mathbf{v}_{\text {ref }, 0}$ is a first or prior guess for reference velocity. In this paper, we choose $\mathbf{v}_{\text {ref, } 0}=0$ near the bottom, but other choices are equally possible. The vertical position of the reference velocity can be different for each station pair and no assumptions about a hypothetical level-of-no-motion are made. The weight matrices $\mathrm{W}_{r}^{(T, S, v)}$ can be horizontally variable; in Section 4 they are chosen to be diagonal with diagonal elements $(\Delta x / 0.01)^{2}$, where $\Delta x=\Delta x(x)$ is the local horizontal grid spacing.

Smoothness could also be imposed by penalizing the curvature (second derivatives) of the hydrographic fields [e.g., Nechaev and Yaremchuk, 1995; Losch et al., 2002]. However, in the present finite element framework, this choice requires higher order basis functions (see Section 3), which we want to avoid for simplicity.

The optimal solution for the (independent) control parameters $T, S$, and $v_{\text {ref }}$ minimizes objective function (2), subject to equation (1). This solution is found by an iterative procedure, in our case a BFGS quasi-Newton algorithm with bounds as implemented by Kelley [1999]. The BFGS algorithm needs the gradient of the objective function with respect to the control parameters to find the descend direction. Because all operations in the above equations are linear, except for the computation of density from temperature and salinity, constructing this gradient is straightforward.

The Hessian matrix $\mathrm{H}$ of second derivatives of $\mathcal{J}$ can be interpreted as the inverse of the error covariance matrix of the control parameters [Thacker, 1989]. For any derived quantity $\varphi$ that can be expressed as a linear or linearized function of the control parameters $b=\left(T, S, v_{\text {ref }}\right)$ near the solution $b_{0}: \varphi(b)=\varphi\left(b_{0}\right)+\mathcal{L}_{\varphi}\left(b-b_{0}\right)$, with the linear operator $\mathcal{L}_{\varphi}=\left.\varphi^{\prime}\right|_{b_{0}}$, the error covariance can be computed from the Hessian matrix as

$$
\operatorname{cov}(\varphi)=\mathcal{L}_{\varphi}^{T} \mathrm{H}^{-1} \mathcal{L}_{\varphi}
$$

\section{Discretization of the Model Equations}

Clearly, the discretization involves only that of linear operations. We choose a finite element grid that is based on a Delaunay triangulation [Barber et al., 1996]. Figure 1 shows an example of such a grid for a section across the Fram Strait between Spitsbergen and Greenland in the Arctic Ocean (inset figure in Figure 1). Note that the grid is almost regular, that is, the grid nodes are aligned in both the horizontal and the vertical. Only near the bottom are some triangles slanted to resolve the topography. But the inclination of these triangles is much smaller than it appears to be from the figure, because in the figure, as usual, the depth is scaled up by orders of magnitude. Nevertheless, the inclination of these triangles can cause errors in the horizontal gradient computation, and their inclusion must be considered carefully.

\subsection{Finite Elements}

Following the general procedure in finite element methods, the thermal wind equations are rewritten in weak form, that is, they are projected onto appropriate test functions $\tilde{\varphi}$ and integrated over the computational domain:

$$
\iint \frac{\partial v}{\partial z} \tilde{\varphi} d x d z=-\frac{g}{\rho_{0} f} \iint \frac{\partial \rho}{\partial x}(T, S, p) \tilde{\varphi} d x d z .
$$




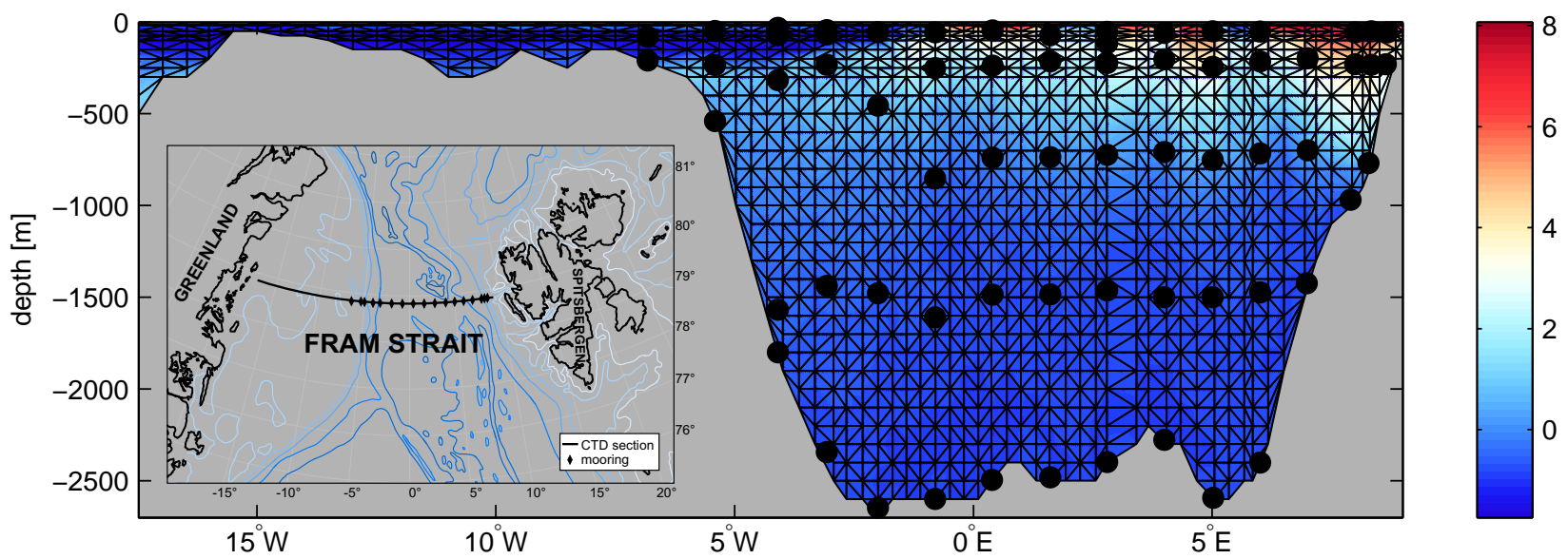

Figure 1. Numerical grid of a section across Fram Strait at approximately $79^{\circ} \mathrm{N}$ with inset map of the area. In color the measured in situ temperature in ${ }^{\circ} \mathrm{C}$ in August 2002. The black dots mark the positions of moored instruments, while the grid nodes are chosen to coincide with the position of hydrographic measurements.

We now describe two different discretizations on the triangular grid in Figure 1: the first approximates all continuous variables by piecewise linear functions, the second uses piece-wise constant functions to represent velocities on the grid and piece-wise linear functions for all other variables.

\subsubsection{Linear basis functions for velocity}

The standard Galerkin method replaces the continuous function $v, \rho$, and $\tilde{\varphi}$ by an expansion into basis functions $\phi_{i}$ that are one at the current node $i$, and zero at all other nodes:

$$
\begin{aligned}
v(x, z) & =\sum_{i} v_{i} \phi_{i}(x, y) \\
\rho(x, z) & =\sum_{i} \rho_{i} \phi_{i}(x, y) \\
\tilde{\varphi}(x, z) & =\sum_{i} \tilde{\varphi}_{i} \phi_{i}(x, y) .
\end{aligned}
$$

$v_{i}, \rho_{i}$, and $\tilde{\varphi}_{i}$ denote point values at grid node $i$. After substitution, equation (5) becomes

$$
\begin{aligned}
& \sum_{i, j} v_{i} \tilde{\varphi}_{j}\left(\iint \phi_{j} \frac{\partial \phi_{i}}{\partial z} d x d z\right)= \\
&-\frac{g}{\rho_{0} f} \sum_{i, j} \rho_{i} \tilde{\varphi}_{j}\left(\iint \phi_{j} \frac{\partial \phi_{i}}{\partial x} d x d z\right)
\end{aligned}
$$

with the matrices

$$
\begin{aligned}
U_{i j} & =\iint \phi_{j} \frac{\partial \phi_{i}}{\partial z} d x d z \\
R_{i j} & =-\frac{g}{\rho_{0} f} \iint \phi_{j} \frac{\partial \phi_{i}}{\partial x} d x d z .
\end{aligned}
$$

Equation(7) holds for any $\tilde{\varphi}$. Thus, the equivalent problem to equation(7), in matrix notation, is

$$
\mathrm{U} \mathbf{v}=\mathrm{R} \rho, \text { or } \mathbf{v}=\mathrm{U}^{-1} \mathrm{R} \rho \text {. }
$$

The boundary condition at the bottom $z=-h, v(-h)=v_{\text {ref }}$, is applied by replacing in matrix $\mathrm{U}$ the row corresponding to the boundary node with $(0, \ldots, 1, \ldots, 0)$, where the one is the diago- nal element $U_{i i}$, and moving the boundary value $v_{\text {ref }}$ to the right hand side. This also ensures the existence of the inverse of $U$.

The basis functions $\phi_{i}$ are chosen as piece-wise linear functions on all elements that belong to grid node $i$ :

$$
\phi_{i}(r)= \begin{cases}1-r & \text { for all elements that contain } \\ & \text { node } i \\ 0 & \text { for all other elements. }\end{cases}
$$

$r$ is the distance from node $i$ normalized by the distance between node $i$ and the neighboring node. With this definition, matrices $\mathrm{U}$ and $\mathrm{R}$ are readily computed.

\subsubsection{Piece-wise constant basis functions for velocity}

So far, we have computed velocities at the same points, the grid nodes, that also represent the temperature and salinity measurements. In contrast, the standard dynamic method computes velocities between station pairs. To achieve a similar numerical scheme, the Galerkin equations (5) require a small modification. Partial integration of the left hand side of equation(5) in $z$ yields

$$
\begin{aligned}
\int v \tilde{\varphi}(\mathbf{n} \cdot \mathbf{z}) d x-\iint & v \frac{\partial \tilde{\varphi}}{\partial z} d x d z= \\
& -\frac{g}{\rho_{0} f} \iint \frac{\partial \rho}{\partial x}(T, S, p) \tilde{\varphi} d x d z,
\end{aligned}
$$

where the first integral is taken along the boundaries at the surface and at the bottom of the domain; $(\mathbf{n} \cdot \mathbf{z})$ is the projection of the normal to the boundary onto the vertical. After specifying boundary values for the velocity $v$ at either surface or bottom, the value of the first integral in equation(12) along the other boundary follows from the so-called solvability condition for weak solutions. For example, assume that boundary values for velocity $v$ are imposed at the bottom. Then $\int v \tilde{\varphi} d x$ over the surface is computed as follows: the second term in the left side of equation (12) vanishes if $\tilde{\varphi} \equiv 1$, and the surface integral must balance the integral over the bottom and the right hand side integral in equation(12).

Instead of this procedure we replace $v$ with a potential $V$, such that

$$
v=\frac{\partial V}{\partial z}
$$

and solve for $V$ instead of $v$. The resulting second-order differential equation for the new unknown variable $V$ requires two boundary conditions: one is the reference velocity and the other is an arbitrary constant that does not affect the velocity $v$. For example, we can choose a $V(z=0)$ at the surface so that the first term in equation(12) represents the boundary integral along the bottom boundary 
only. $V$ is represented with the same linear basis functions $\phi_{i}$ as density and the test functions $\tilde{\varphi}$. As a consequence, $v$, since it is the derivative of $V$, is now represented by basis functions that are piecewise constant on the elements.

\subsection{Testing the Discretized Thermal Wind Equations in Idealized Geometries}

\subsubsection{Front over a steep slope}

In a first test of the finite element discretization, we compute velocities from thermal wind relative to the bottom or the surface through an idealized domain with a front over a steep slope (Figure 2). We assume a density field that is an inverse tangent function of the horizontal coordinate: $\rho=[\arctan (a x+b)+c z] \mathrm{kg} \mathrm{m}^{-3}$, so that we can integrate equation (1) analytically. For our choice of density with $a=1 /(50 \mathrm{~km}), b=20, c=-3 /(400 \mathrm{~m})$, a domain that is $1000 \mathrm{~km}$ wide and has a maximum depth of $400 \mathrm{~m}$, and with $g /\left(\rho_{0} f\right)=100 \mathrm{~m}^{4} \mathrm{~kg}^{-1} \mathrm{~s}^{-1}$, we obtain a transport of $5.60 \mathrm{~Sv}$ $\left(1 \mathrm{~Sv}=10^{6} \mathrm{~m}^{3} \mathrm{~s}^{-1}\right)$.

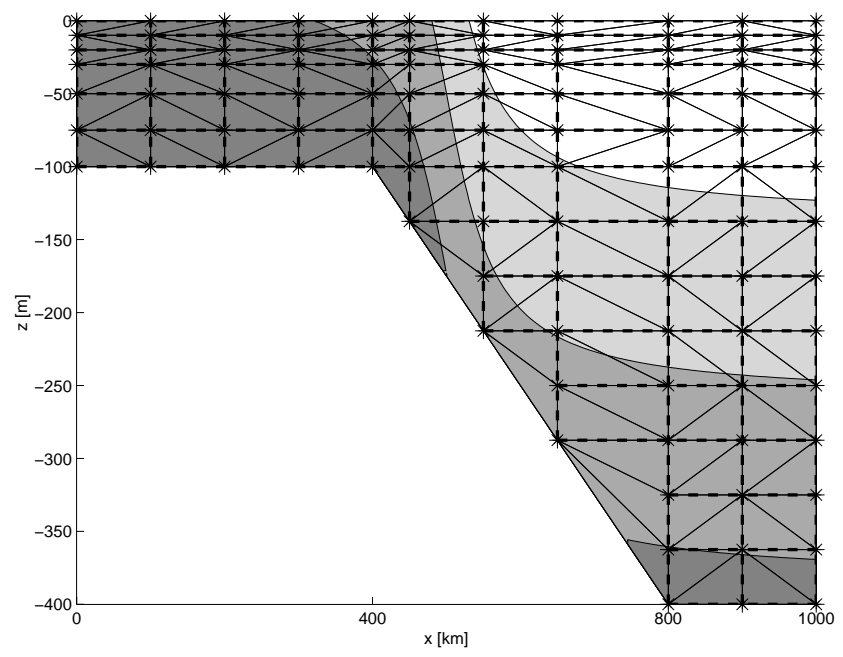

Figure 2. Test domain with hypothetical measurement points (stars). Solid thin lines mark the finite element triangular grid, the thick dashed lines correspond to the finite difference grid of the dynamic method. The shading shows the density front with dark shades for dense water and light shades for light water.

The standard dynamical method, for example, as implemented in the matlab routines of the CSIRO sea water library [Morgan, 1994], computes geopotential height anomalies at the location of vertical casts of temperature and salinity. Velocities are then calculated between station pairs and therefore, the method is accurate to first order. However, because of the finite resolution in the horizontal, special interpolation techniques are required near the bottom to resolve bottom wedges [e.g., Wunsch, 1996]. Ganachaud [2003] compared different interpolation methods and found that they can yield transports that are different by several Sverdrups. Assuming that the transport through the bottom wedges vanishes the dynamic method gives a transport of $4.85 \mathrm{~Sv}$, relative to the bottom, if measurements of temperature and salinity are available for the hypothetical stations in Figure 2. In a second case the reference level is chosen to be at the surface, that is, the largest velocities occur near the bottom. In this case, which serves as an extreme case only, the dynamic method is even less accurate and yields a transport of $4.05 \mathrm{~Sv}$.

Our finite element discretization is based on piece-wise linear basis functions for density (or temperature and salinity) and either piece-wise linear or constant basis functions for velocity. If the density field is chosen to vary linearly between stations these methods are exact.

However, for our density field that is a non-linear function of the horizontal coordinate, we get deviations from the exact transport

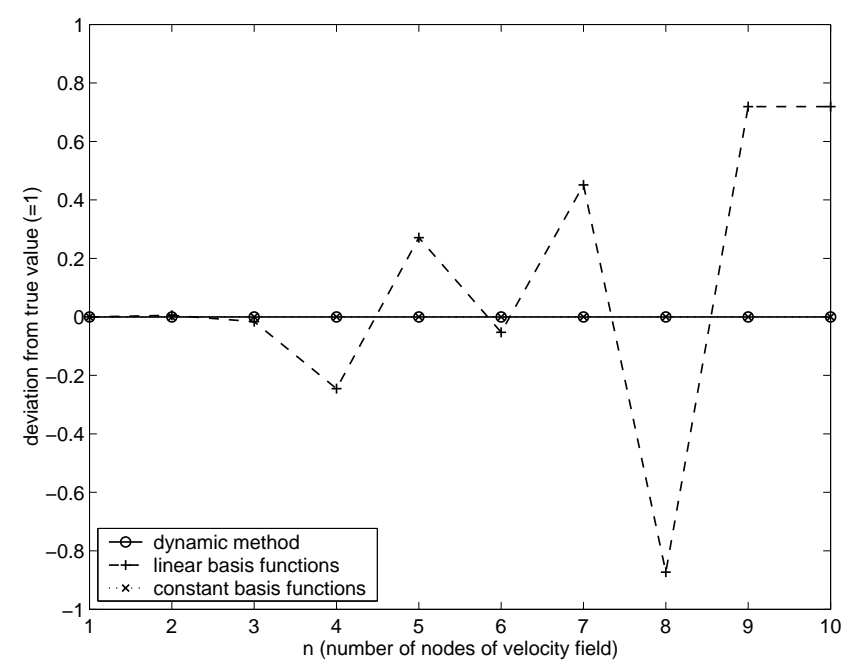

Figure 3. Deviation of transport values from the true value of 1 as a function of number of velocity nodes for three different methods.

by $0.04 \mathrm{~Sv}$ for piece-linear basis for velocity and and $-0.17 \mathrm{~Sv}$ for piece-wise constant velocity basis functions. Also in the extreme case of zero reference velocities at the surface the estimated transport is much more accurate than for the finite-difference method: for piece-wise linear velocity basis functions we get $5.30 \mathrm{~Sv}$ and for piece-wise constant functions $5.77 \mathrm{~Sv}$.

\subsubsection{Flat bottom}

In a different test case, we test the performance of piece-wise linear versus piece-wise constant basis functions for velocity. For this, geostrophic velocities relative to the bottom are computed in a domain with a flat bottom at depth $h=\sqrt{2}$ and length $L=1$. The density field is sinusoidal with the horizontal coordinate: $\rho=\rho_{0}+\frac{\rho_{0} f}{g} \cos \frac{2 n-1}{2 L} \pi x$, where $L$ is the length of the section and $n=1,2^{g} \ldots$ is the number of velocity nodes. For a flat bottom the horizontal and vertical integrations to compute the total transport are independent and the transport only depends on the net density difference across the section, which is the same for all $n$, so that the analytical transport is always $\frac{1}{2} h^{2}=1$. Ten hypothetical stations with constant spacing define the horizontal grid. The example is constructed so that central differences yield exact results, because in summing up the differences between the values at grid node $i$ and $i-1$ cancels all interior contributions exactly. Consequently, the dynamic method yields a transport value 1 independent of $n$ (Figure 3). For the finite element method with piece-wise linear basis functions for velocity the transport deviates from the correct value as the resolution decreases relative to the number of velocity nodes $n$. On the other hand, the transport values obtained from finite elements and piece-wise constant basis functions for velocity are also indistinguishable from the true value 1 . In fact, deviations are smaller than $10^{-14}$ and therefore within machine precision.

For this problem, the highest accuracy is achieved with central differences, because the horizontal operator acting on density is first order, while the velocity is subject to a zero order differential operator in the horizontal. Therefore discretizing density with a higher order $($ linear $=$ first order) basis function than velocity $($ constant $=$ order zero) results in a more accurate transport value than using the same order (linear) for both density and velocity. Consequently piece-wise constant basis functions for velocity are preferred for this application.

\section{A First Application: Fram Strait}

Fram Strait represents the only deep connection between the Arctic Ocean and the Nordic Seas. This connection is two-fold: Arctic 
Ocean freshwater from the north has a strong impact on convection in the Nordic Seas and further south, while warm and saline Atlantic waters from the south affect the water mass characteristics in the Arctic Ocean and influence ice and atmosphere. The topographic constraints of the Fram Strait split the West Spitsbergen Current (WSC), which carries Atlantic Water northward, into at least three branches. Two of them enter the Arctic Ocean along different pathways. The third branch recirculates immediately in the Fram Strait. The East Greenland Current (EGC) carries water from the Arctic Ocean southwards in a concentrated core above the continental slope.

The variability of oceanic fluxes through the Fram Strait has been monitored by an exceptionally dense array of moorings since 1997 [Fahrbach et al., 2001; Schauer et al., 2004]. Timeseries of temperature and velocity from moored instruments provide estimates of heat and volume fluxes with excellent temporal resolution but the spatial structure of the flow is under-resolved. This is the main source of the error in previous transport estimates. With our new finite element section inverse model, we expect to reduce the problem of spatial resolution-because we can now use additional hydrographic data that has a higher spatial resolution than the moorings - and thus, obtain more reliable transport estimates by combining all available data.

\subsection{Velocity Measurements and Hydrographic Data}

The array of current meter moorings is described comprehensively in Woodgate et al. [1998]; Fahrbach et al. [2001] and Schauer et al. [2004]. We only repeat a few details that are relevant here: The mooring array extends from the eastern Greenland shelf break to the western shelf break off the coast of Spitsbergen (Figure 1). From 1997 to 2001, 14 moorings covered the section, except for the years 1999 and 2000 when 3 moorings in the central part of the strait were not deployed. In 2002 the array was augmented with 2 additional moorings in the recirculation area between 0 and $2{ }^{\circ} \mathrm{E}$ and one at the Greenland shelf. The horizontal spacing of the moorings increases over the continental slopes. The instruments are deployed at depths starting at $10 \mathrm{~m}$ above the seabed to approximately $60 \mathrm{~m}$ below the surface. Figure 4 shows the position of the moorings in August 2002. Each year during redeployment of the array in summer or autumn, hydrographic measurements were carried out at CTD-stations along the mooring line with high spatial resolution. In order to exploit the flexibility of the finite element method, we use the positions of the CTD-stations to generate the grid, so that the interpolation from model variables to data is trivial for temperature and salinity.

Without the hydrographic section data, the largest uncertainty of transport estimates from moored current meters and temperature recorders stems from aliasing due to the low resolution of the available data points. In previous work, measurements of moored instruments were mapped to a fine resolution grid by kriging [e.g., Deutsch and Journel, 1992] to yield temperature and absolute velocity fields that resolve the warm core of the West Spitsbergen Current and the shallow flow of the East Greenland Current rather well when compared to temperature measurements or relative geostrophic velocity fields from high resolution CTD sections [Schauer et al., 2004]. However, the complex structure of the return flow in the central Fram Strait is not properly resolved by the moored array. Further, mesoscale eddies are aliased into large scale flow by distant moorings [Fahrbach et al., 2003]. Temporal averaging into monthly means of measured velocities eliminates transient small scale structures, but stationary mesoscale eddies over topography, which are observed in the central Fram Strait, remain aliased.

\subsection{Weight Matrices and Prior Error Estimates}

Prior to inversion of all data with the help of the finite element model FEMSECT, weighting matrices for equations (2) and (3) have to be constructed. The weighting matrices define the "physically acceptable" deviations of the model state from data and constraints. They are taken to be the inverses of error covariances that are estimated prior to the inversion, so that a larger prior error amounts to a smaller weight and less allowed deviation from the corresponding

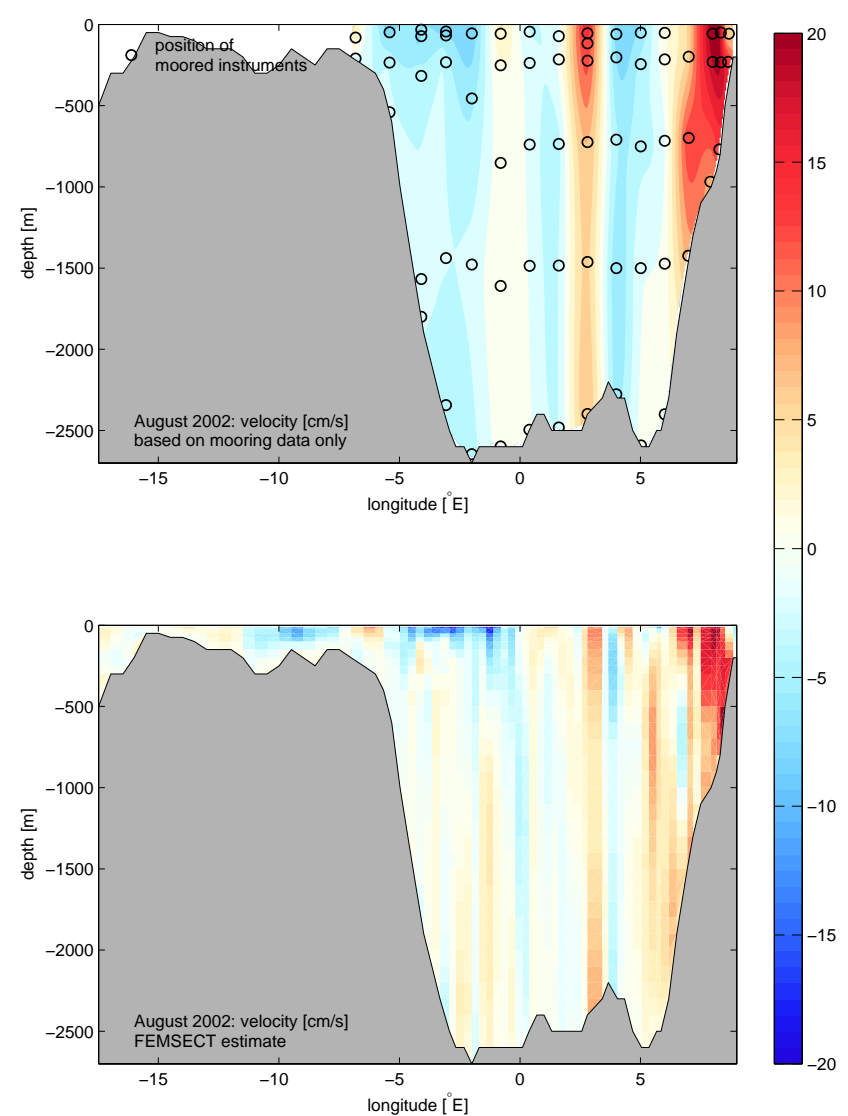

Figure 4. Velocities estimated by interpolating moored instrument data (top, with position of moored instruments) and estimated with the help of the inverse model FEMSECT (bottom).

datum and vice versa. With the exception of $T$ and $S$ from CTD measurements all cross-correlations are neglected and the covariance matrices are diagonal.

For $T$ and $S$ from CTD-measurements, we construct covariances-local to the respective CTD-station-from vertical correlation and cross-correlations between $T$ and $S$. This procedure, which follows Losch et al. [2002], allows the model to change the depth of an isopycnal without changing the $T$ - $S$ relation on the isopycnal [Yaremchuk et al., 2001]. To these covariances, we add a depth dependent estimate of uncorrelated "ocean noise" according to Bindoff and McDougall [1994], that is, the variance of differences between neighboring stations, and an estimate of uncorrelated "measurement error". The latter can include both actual instrument errors and possible errors that arise from the quasi-synopticity of the CTD-data. Here, we allow a large error of $1^{\circ} \mathrm{C}$ for $T$ and 0.1 for $S$, because CTD data are often not representative of the entire period covered by the mooring data. In total, the covariance estimate includes diagonal terms on the order of $\left(1^{\circ} \mathrm{C}\right)^{2}$ near the bottom to $\left(1.75^{\circ} \mathrm{C}\right)^{2}$ near the surface for temperature and $0.1^{2}$ to $0.75^{2}$ for salinity, respectively; off-diagonal terms are smaller.

For velocity data we assume an uncorrelated error of $1 \mathrm{~cm} \mathrm{~s}^{-1}$, the initial reference velocity is assumed to be $0 \pm 5 \mathrm{~cm} \mathrm{~s}^{-1}$. We impose smoothness by specifying $\mathrm{W}_{r}$ in equation(3) to be $(\Delta x / 0.01)^{2}$ for both $T, S$, and velocity $v$, where $\Delta x=\Delta x(x)$ is the local horizontal grid spacing.

In order to reduce the amount of hydrographic data, we interpolated the $1 \mathrm{dbar}$ data to standard depth levels $(-10 \mathrm{~m},-20 \mathrm{~m},-50 \mathrm{~m}$, $-75 \mathrm{~m},-100 \mathrm{~m},-150 \mathrm{~m},-200 \mathrm{~m},-250 \mathrm{~m},-300 \mathrm{~m},-400 \mathrm{~m},-500 \mathrm{~m}$, etc.) to obtain 1404 data points for temperature and salinity each for the 72 stations of CTD-section in August 2002. In that year there were 

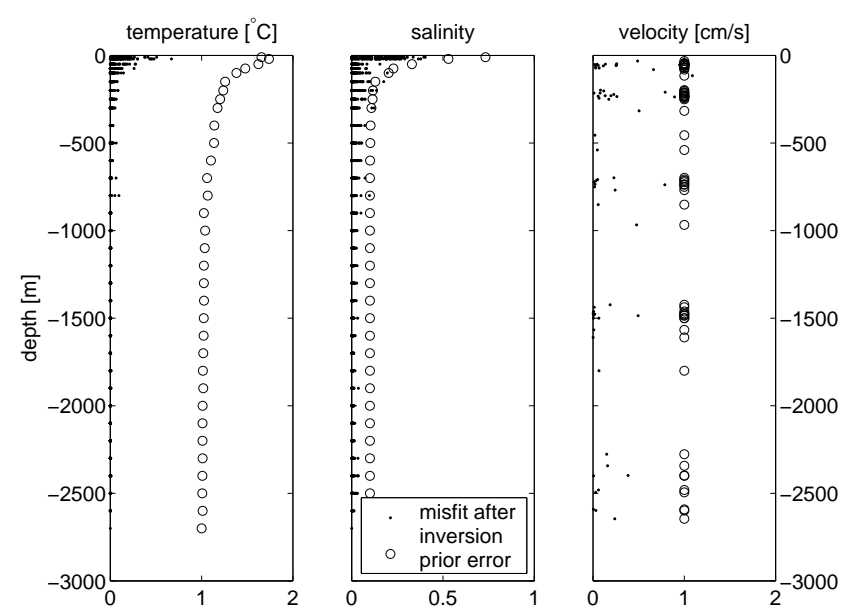

Figure 5. Model-data misfit after inversion (dots) with prior errors (circles) for August 2002 (Figure 4), for temperature (left), salinity (middle), and velocity (right) data. Only near the surface a few model-data misfit values remain are larger than the prior errors, so that for the most part the assumption of geostrophic balance is valid.
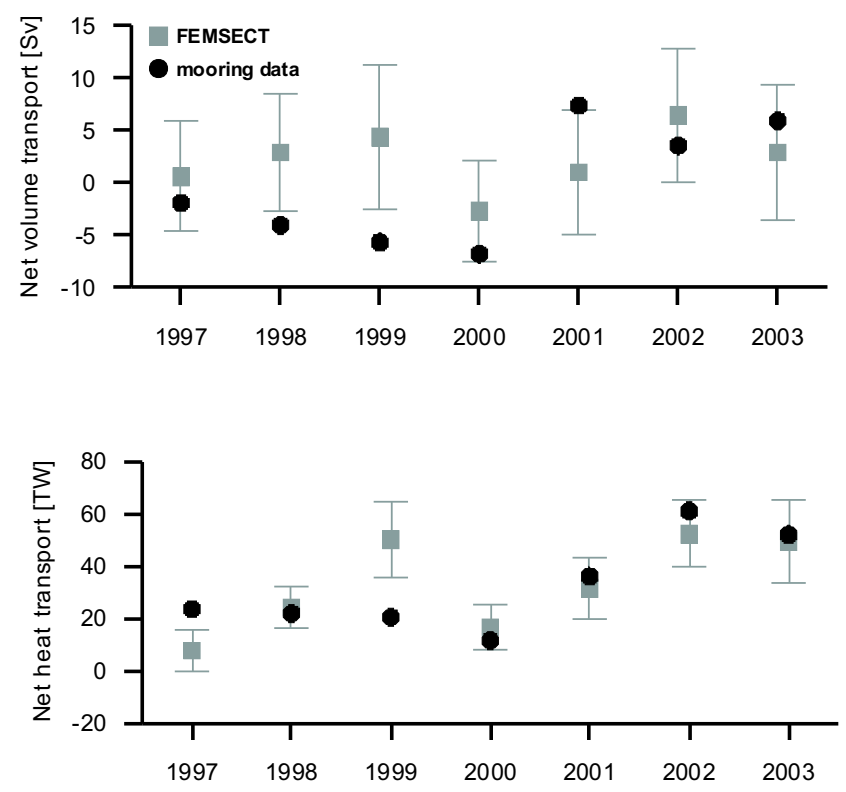

Figure 6. Transport estimates for 7 different years. The estimates do not always refer to data from the same month of the year and should therefore not be treated as a timeseries. The differences in transports between 1998 and 2001 can be explained by the resolution of the mooring array that is too coarse to represent the recirculation patterns in the central Fram Strait. FEMSECT can reduce this resolution problem by using additional information from CTD-data and geostrophy.

66 moored current meters in place. The flexibility of the finite element grid allows to choose the grid nodes to coincide naturally with the hydrographic data points, so that the dimension of the control vector is $2 \times 1404+71=2879$. For other years the number of data and grid points is comparable. With this configuration we needed typically on the order of 1000 iterations to reach convergence, measured by a reduction of the norm of the gradient by a factor of 1000 . a)

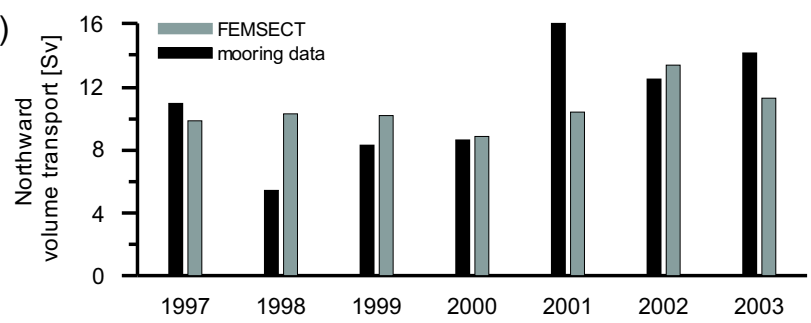

b)

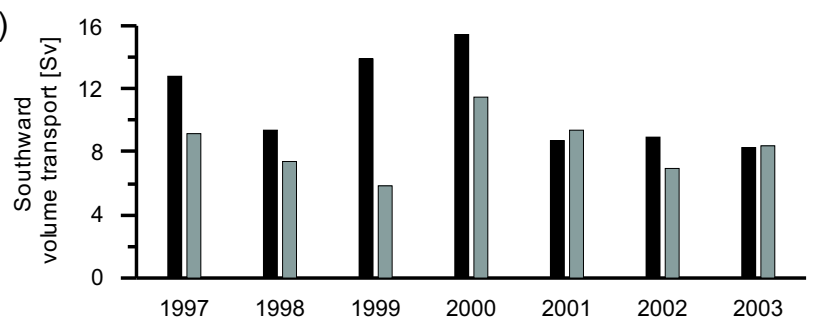

Figure 7. Comparison of northward and southward volume transports between FEMSECT and the mooring data alone in $\mathrm{Sv}\left(1 \mathrm{~Sv}=10^{6} \mathrm{~m}^{3} \mathrm{~s}^{-1}\right)$.

\subsection{Velocity Fields and Transport Estimates}

Velocity from interpolation of mooring data by kriging and from a full inversion of all CTD and mooring data with FEMSECT are shown in Figure 4 for August 2002. Both velocity fields reveal an intense northward flow of the West Spitsbergen Current, occupying the whole water column at the eastern shelf slope. Measurements by moored current meters in the top panel of Figure 4 clearly show its strong barotropic character. In this area, the inverse solution in the bottom panel differs only slightly from the kriging solution by additional (geostrophic) shear at the lower boundary of the Atlantic water layer at approximately $700 \mathrm{~m}$ depth. At the opposite side of the Fram Strait, the shallower and weaker East Greenland Current shows up as a southern flow with a core over the Greenland shelf slope. The strong density gradient in the upper layer gives rise to significant vertical velocity shear which is much better reproduced by the inverse solution than by the velocity obtained from moorings alone; the latter tend to have smooth vertical velocity gradients (small vertical shear) due to the interpolation. A part of the Atlantic water recirculates in the central Fram Strait and the velocity field from the inversion suggests that this flow is characterized by smaller spatial scales than are resolved by the mooring array.

Temperature and salinity are control variables of FEMSECT and are adjusted during the inversion. Figure 5 shows the model-data misfit after the inversion (dots) along with the prior errors (circles) as a function of depth. Generally, the model-data misfit is consistent with the prior errors. The resulting difference between initial and inverted density field can reach $0.3 \mathrm{~kg} \mathrm{~m}^{-3}$ (not shown). The difference is most pronounced in areas with strong vertical density gradients, especially in the western part of the strait. Over the Greenland shelf this density difference is associated with the freshwater layer in the upper $30-50 \mathrm{~m}$. Over the continental slope density differences are large in the core of the East Greenland Current because the inverse model changes the density field in order to fit a geostrophic shear to the current measurements. Model velocity deviates from the moored instrument data by less than the prior error of $1 \mathrm{~cm}$ after the inversion except for near surface values.

In Figure 6 we compare estimates of volume transports and heat transports relative to $-0.1^{\circ} \mathrm{C}$ through the Fram Strait obtained with the kriging method described in Schauer et al. [2004] from monthly averages of current and temperature data from moored instruments for seven different times during the period 1997-2003, to estimates of the inverse model FEMSECT from the same data plus hydrographic data from the CTD section. (The heat transports 
Table 1. Transport estimates for the two sensitivity experiments with complete and reduced mooring data sets.

\begin{tabular}{|c|c|c|c|c|}
\hline & $\begin{array}{c}\text { Volume net } \\
{[\mathrm{Sv}]}\end{array}$ & $\begin{array}{c}\text { Volume } \\
\text { northward [Sv] }\end{array}$ & $\begin{array}{c}\text { Volume } \\
\text { southward }[\mathrm{Sv}]\end{array}$ & Heat net $[\mathrm{TW}]$ \\
\hline \multicolumn{5}{|l|}{ August 2002} \\
\hline Mooring data only & 3.6 & 12.5 & -9.0 & 61.2 \\
\hline Mooring data only, with gap & 15.5 & 23.4 & -7.9 & 70.4 \\
\hline FEMSECT for CTD section with complete moorings array & $6.4 \pm 6.4$ & $13.4 \pm 4.3$ & $-7.0 \pm 3.3$ & $52.4 \pm 12.7$ \\
\hline FEMSECT for CTD section with moorings array, with gap & $6.9 \pm 6.7$ & $13.4 \pm 4.4$ & $-6.5 \pm 3.5$ & $52.5 \pm 12.8$ \\
\hline \multicolumn{5}{|l|}{ September 2003} \\
\hline Mooring data only & 5.9 & 14.2 & -8.3 & 52.1 \\
\hline Mooring data only, with gap & 12.2 & 20.1 & -7.9 & 56.6 \\
\hline FEMSECT for CTD section with complete moorings array & $2.9 \pm 6.5$ & $11.3 \pm 4.2$ & $-8.4 \pm 3.4$ & $49.7 \pm 15.8$ \\
\hline FEMSECT for CTD section with moorings array, with gap & $3.2 \pm 6.8$ & $10.7 \pm 4.6$ & $-7.5 \pm 3.4$ & $48.5 \pm 16.0$ \\
\hline
\end{tabular}
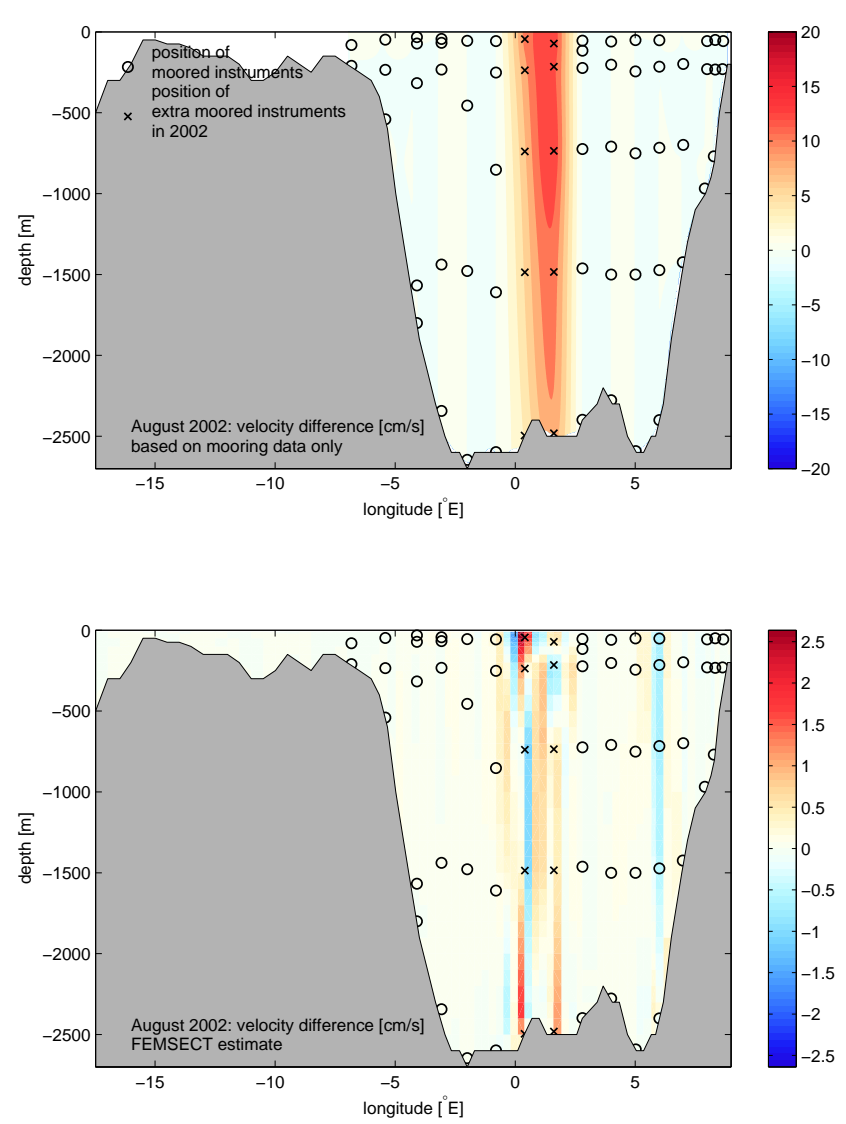

Figure 8. Velocity difference between solutions based on the complete and the reduced mooring data set for August 2002. Top: interpolation of moored instrument data, bottom: inverse solution with moored instrument data and CTD data. Note, that the color scale is different by one order of magnitude.

are strictly speaking temperature transports, as the mass balance through the Fram Strait is non-zero, but we stick to the jargon of relative heat transports for convenience.) In all cases, we assume quasi-synopticity and only use moored data from the months during which CTD data are available, because for now we wish to avoid mixing data from different time periods for simplicity.

FEMSECT provides uncertainty estimates along with the solution. These uncertainty estimates are computed from the inverse Hessian of the objective function $\mathcal{J}$ in equation (2). In the limit of Gaussian statistics, the inverse Hessian can be interpreted as the error covariance matrix of the control parameters temperature, salinity, and reference velocity (cf. Section 2.2). The estimated uncertainties then correspond to the standard deviation of a normally distributed variable. With our choice of prior error estimates, the volume transport uncertainties are of the order of $6-7 \mathrm{~Sv}$, that is, in some cases larger than the volume transport estimate itself. It is important to understand that the simultaneous inversion of all data and model equations results in a formal error propagation that translates the measurement and model errors into uncertainties of estimated quantities such as net volume transport. For example, we chose a prior error of $5 \mathrm{~cm} \mathrm{~s}^{-1}$ for the bottom (reference) velocities. Changing this prior error to $1 \mathrm{~cm} \mathrm{~s}^{-1}$ reduces the estimated uncertainty by $14 \%$; reducing the prior "measurement errors" for temperature $\left(1^{\circ} \mathrm{C}\right)$ and salinity $(0.1)$ by a factor of five, decreases the posterior uncertainty by $35 \%$ of the previous value. As a more radical test, we increased the weight of all regularization terms by a factor of 4 , that is, doubled the prior errors for reference velocity and roughness, to obtain posterior volume and heat transport errors that are increased by $40-50 \%$. Thus, the size of the estimated uncertainty of inverse solutions is controlled by the prior errors that define the weights in the objective function. It is the responsibility of the investigator to check that both prior and posterior errors are consistent with the model solution and observations Wunsch [1996].

For the years 1998 to 2001, net volume transport estimates from the different methods differ by as much as the uncertainty estimate provided by FEMSECT. Separating the volume transport into a northward and a southward component (integral over all northward and southward velocities, respectively) points towards an explanation that relates the large difference to the resolution of the mooring array (Figure 7): In the years 1997, 1999, and 2000, the estimated southward volume transport is larger with the interpolation method than with FEMSECT while the northward transports are similar for both methods. In contrast, the southward transports are similar in 1998 and 2001, but now the northward transport estimates are off by as much as $4 \mathrm{~Sv}$. All years up to 2001 have in common that the mooring array had a gap between $1^{\circ} \mathrm{W}$ and $3^{\circ} \mathrm{E}$ which leads to a misrepresentation of the recirculation region in the interpolated data. In the years 1997 to 2000, a prevailing southward flow was measured at both ends of the gap, which gives enormous southward transport when interpolated across the significant distance of the gap. A change in the circulation pattern in 2001 results in the opposite effect: Northward flow measured at the ends of the gap and interpolated across the gap results in the strongly overestimated northward volume transport and in consequence in a net transport to the north. FEMSECT uses additional data and geostrophic velocity information instead of simply interpolating mooring data. By combining the mooring data with hydrographic data and geostrophy, FEMSECT can provide reliable and realistic velocity and transport estimates that are superior to those obtained from unphysical interpolation of data.

This problem of unresolved recirculation between $-1^{\circ} \mathrm{W}$ and $3^{\circ} \mathrm{E}$ was recognized [Fahrbach et al., 2003] and resolved by deploying additional moorings in the recirculation region in 2002 and 2003. With the additional data, a much more realistic representation of the flow field in the central Fram Strait is obtained (BeszczynskaMöller et al., Variability of volume and heat fluxes through Fram Strait from the array of moorings, manuscript in preparation). Consequently, transport estimates from mooring data alone and inverse estimates agree much better in those years. 
The net heat transport relative to $-0.1^{\circ} \mathrm{C}$ is less sensitive to the resolution problem described above than the net volume transport. This observation supports the above hypothesis of an unresolved flow field in the center of the strait, because almost all heat (i.e., temperature relative to $-0.1^{\circ} \mathrm{C}$ ) is carried north by the West Spitsbergen Current, that is, by a permanent feature in the eastern part of the strait that is also well resolved by the mooring array. Therefore, the estimates based on mooring data alone and FEMSECT estimates generally agree with each other quite well. With one exception (in 1999 when the volume transport estimates also differ most), all estimates of net heat transport from moorings fall within the uncertainty range of FEMSECT estimates (Figure 6). For 2002 and 2003, the net volume transports estimated from moored instrument data also fall into the error range estimated with FEMSECT.

In the following, we test the hypothesis that better spatial resolution of data improves transport estimates from interpolated mooring data and that the lack of data resolution explains the larger difference between interpolation and inverse model solution. From two mooring data sets from the period with increased spatial coverage by moorings in the central Fram Strait (August 2002 and September 2003), the central two moorings are removed to mimic the situation of previous years. Figure 8 compares velocity fields obtained from interpolation of all moored instrument data and the inverse model to the respective solutions for the reduced mooring data set for August 2002. If mooring data is interpolated to yield velocity fields, removing the two moorings in the center of the strait (open circles) leads to a dramatically different velocity field in the central part of the section. Northward velocity differences over $10 \mathrm{~cm} \mathrm{~s}^{-1}$ are clearly a result of the poor resolution. On the other hand, the structure of the currents reproduced by FEMSECT seems to be much less sensitive to the spatial resolution of the mooring data.

Table 1 shows the corresponding volume and heat transport estimates for August 2002 and September 2003. For both periods removing the two central moorings results in a strongly increased northward volume transport for the kriging method: The estimate is nearly doubled for August 2002 and 50\% higher for September 2003. Together with a much weaker decrease of the southward volume transport, overestimating the northward component yields a net volume transport that is almost three times larger in 2002 and two times larger in 2003. At the same time, net volume transport estimates by the inverse model, based on the complete and the reduced mooring data set are different by less than $10 \%$ in both cases. Differences in estimated heat transports are also of one order of magnitude larger in the case of estimates based on mooring data alone as compared to inverse model results.

While we would like to claim that our inverse solution is far superior to the interpolation method, it should be noted that this positive result is to some extent fortuitous: Inspection of Figure 4 reveals that at the two locations of the additional moorings the measured velocities near the bottom are small, so that our prior estimate of zero bottom velocities is justified $a$ posteriori. Together with a realistic bottom velocity, the geostrophic shear provides a reasonable velocity estimate throughout the water column, which does not need correction by measurements of absolute velocities (by moored instruments). However, adding more data always improves the inverse solution by decreasing the formal posterior error estimate (Table 1).

We emphasize that the choice of zero bottom velocity is by no means a limitation of the method: any prior estimate of bottom velocities can be used in the inverse model.

\section{Conclusion}

Inverse methods and finite elements are a perfect combination for analyzing irregularly spaced section data in oceanography. With the help of inverse methods, data of different type and resolution can be combined with dynamical balances to yield a dynamically consistent best fit to all available information. Finite elements, on the other hand, provide an elegant and consistent way to account for irregularly spaced data and complex topography with a triangular grid.
Grid nodes can be chosen to coincide with data locations making interpolation of model variables to data trivial. Also, the finite element method requires the numerical formulation of the dynamical equations in matrix form, which in turn simplifies the formulation of the adjoint model for the gradient computation: the adjoint of a matrix operator is simply its transpose.

All these characteristics of finite elements and inverse methods can be combined to formulate a new analysis tool for oceanographic section data. This model, which we call FEMSECT for Finite Element Method inverse SECTion model, is used to estimate transports of volume and heat through the Fram Strait, where an exceptional data set with moored instrument data and hydrographic data from oceanic transects is available. Combining all data already reveals that calculations from interpolation of mooring data alone tend to overestimate total transports in the case of poor spatial resolution of moored instrument data. FEMSECT provides more reliable estimates that are less sensitive to the spatial resolution of the moorings because it replaces interpolation with geostrophic shear information from CTD-measurements.

Further analyses of the Fram Strait data are underway. There we investigate time series of moored instrument data for which CTD data are not available at the same time. In this case, the prior errors of the CTD need to be increased to account for the assumption that the density field is constant.

In general, additional data could be included in the analysis of hydrographic sections with FEMSECT. In the absence of moored instruments, ship-based ADCP (Acoustic Doppler Current Profiler) velocity data could fill the gap and provide an estimate of the absolute velocity to yield transport estimates for the entire section.

Further developments of FEMSECT include using basis functions of arbitrary (higher) order in order to increase the accuracy of the solution. With higher-order basis functions it is also natural to include regularization terms in the objective function that penalize the second instead of the first derivative of the fields. In some applications it may be necessary to be able to put the reference velocity at an arbitrary depth within the water column. Currently the position of the reference velocity is restricted to either the bottom or the surface of the domain, so that there is also room for improvement.

The finite element method is very appealing from a mathematical point of view. Especially integral quantities, such as integrated transports through a section, benefit from the conservative properties of the numerical scheme. Together with the geometrical flexibility of a triangular grid, we achieve with our finite element section model a proper representation of the bottom topography and bottom wedges even with limited horizontal resolution; the model provides natural interpolation rules for irregularly spaced data that emerge from the method itself; the model integrates the thermal wind equation accurately if piece-wise constant basis functions are chosen to represent velocity. Piece-wise linear basis functions should only be used for temperature, salinity, and density values for this application.

In order to complement the flexibility of the finite element model, FEMSECT is available as a highly portable Matlab code. It can be obtained from authors on request. More convenient download methods are in preparation. All computations in this paper were performed on ordinary P4 desktop computers, Unix Workstations, and Apple computers. Typically on a $2 \mathrm{GHz}$ P4 with $512 \mathrm{MB}$ RAM, 1000 iterations of the BFGS algorithm took less than 40 minutes for the Fram Strait experiments, and the inversion of the Hessian matrix $\left(\approx 3000^{2}\right.$ elements) less than 1 minute. Note that our problem was sufficiently small to allow a direct inversion of the Hessian matrix in Matlab. Larger problems may require an iterative procedure following Yaremchuk et al. [2001] or Yaremchuk et al. [2002].

When undertaking the difficult task of analyzing sparse data, inverse methods appear the perfect tool to combine information of different origins, such as, in our example, ship-borne measurements, measurements from moored arrays, and dynamical balances. These methods provide both a "best estimate" from data with prior error estimates and a posterior formal error estimate. We conclude that inverse methods should be used routinely for the analysis of data. 
Acknowledgments. The authors would like to thank Sergej Danilov, Gennady Kivman, and Nikolai Yakovlev for discussions about the mysteries of finite elements, Ursula Schauer and Gerd Rohardt for deeper insights into what an observationalist expects of an analysis model, and Eberhard Fahrbach, Olaf Klatt, Volker Strass, and Jill Schwarz for further helpful suggestions. This work is dedicated to the memory of Gennady Kivman.

\section{References}

Barber, C. B., D. Dobkin, and H. Huhdanpaa, The quickhull algorithm for convex hulls, ACM Trans. Math. Softw., 22, 469-483, 1996.

Bindoff, N. L., and T. J. McDougall, Diagnosing climate change and ocean ventilation using hydrographic data, J. Phys. Oceanogr., 24, 1137-1152, 1994.

Cisewski, B., G. Budéus, and G. Krause, Absolute transport estimates of total and individual water masses in the Northern Greenland Sea derived from hydrographic and ADCP measurements, J. Geophys. Res., 108, doi:10.1029/2002JC001,530, 2003.

Danilov, S., G. Kivmann, and J. Schröter, A finite element ocean model: Principles and evaluation, Ocean Modelling, 6, 125-150, 2004.

Deutsch, C. V., and A. G. Journel, GSLIB - Geostatistical Software Library and User's Guide, Oxford University Press, New York, 1992.

Dobrindt, U., and J. Schröter, An adjoint ocean model using finite elements: An application to the South Atlantic, J. Atmos. Oceanic Technol., 20, 392-407, 2003.

Fahrbach, E., J. Meincke, S. Østerhus, G. Rohardt, U. Schauer, V. Tverberg, and J. Verduin, Direct measurements of volume transports through Fram Strait, Polar Research, 20, 217-224, 2001.

Fahrbach, E., U. Schauer, and G. Rohardt, How to measure oceanic fluxes from the Atlantic Ocean through Fram Strait, ASOF Newsletter, 1, 3-7, 2003.

Fofonoff, P., and R. Millard, Jr., Algorithms for computation of fundamental properties of seawater, Unesco Technical Papers in Marine Science 44, Unesco, 1983.

Ganachaud, A., Error budget of inverse box models: The North Atlantic, $J$. Atmos. Oceanic Technol., 20, 1641-1655, 2003.

Ganachaud, A., and C. Wunsch, Improved estimates of global ocean circulation, heat transport and mixing from hydrographic data, Nature, 408, 453-457, 2000.

Kelley, C. T., Iterative Methods for Optimization, no. 18 in Frontiers in Applied Mathematics, SIAM, Philadelphia, 1999.

Le Provost, C., F. Lyard, J. Molines, M. Genco, and F. Rabilloud, A hydrodynamic ocean tide model improved by assimilating a satellite altimeterderived data set, J. Geophys. Res., 103, 5513-5529, 1998.

Losch, M., R. Redler, and J. Schröter, Estimating a mean ocean state from hydrography and sea-surface height data with a non-linear inverse section model: Twin experiments with a synthetic data set, J. Phys. Oceanogr., 32, 2096-2112, 2002.
Macdonald, A. M., and C. Wunsch, An estimate of the global ocean circulation and heat flux, Nature, 382, 436-439, 1996.

Morgan, P. P., Seawater, a library of matlab computational routines for the properties of sea water, CSIRO Marine Laboratories Report 222, 29pp, 1994.

Myers, P. G., and A. J. Weaver, A diagnostic barotropic finite-element ocean circulation model, J. Atmos. Oceanic Technol., 12, 511-526, 1995.

Myers, P. G., S. Grey, and K. Haines, A diagnostic study of interpentadal variability in the North Atlantic Ocean using a finite element model, Ocean Modelling, 10, 69-81, 2004.

Nechaev, D. A., and M. I. Yaremchuk, Application of the adjoint technique to processing of a standard section data set: World Ocean Circulation Experiment section S4 along $67^{\circ} \mathrm{S}$ in the Pacific Ocean, J. Geophys. Res., 100, 865-879, 1995.

Rintoul, S. R., South Atlantic interbasin exchange, J. Geophys. Res., 96, 2675-2692, 1991.

Schauer, U., E. Fahrbach, S. Østerhus, and G. Rohardt, Arctic warming through the Fram Strait - oceanic heat transport from 3 years of measurements, J. Geophys. Res., 109, doi:10.1029/2003JC001,823, 2004.

Schlichtholz, P., and M.-N. Houssais, An inverse modeling study in Fram Strait. Part I: Dynamics and circulation, Deep-Sea Research II, 46, 1083 $1135,1999$.

Thacker, W. C., On the role of the Hessian matrix in fitting models to data, J. Geophys. Res., 94, 6177-6196, 1989.

Walters, R. A., A model for tides and currents in the English Channel and southern North Sea, Adv. in Water Resources, 10, 138-148, 1987.

Woodgate, R., U. Schauer, and E. Fahrbach, Moored current meters in the Fram Strait at $79^{\circ} \mathrm{N}$ : preliminary results with special emphasis on the West Spitzbergen Current, Berichte des Fachbereichs Physik 91, AlfredWegener-Institut für Polar- und Meeresforschung, 1998.

Wunsch, C., The North Atlantic general circulation west of $50^{\circ}$ determined by inverse methods, Rev. Geophys. Space Phys., 16, 583-620, 1978.

Wunsch, C., The Ocean Circulation Inverse Problem, Cambridge University Press, Cambridge, 1996.

Yaremchuk, A., M. Yaremchuk, J. Schröter, and M. Losch, Local stability and estimation of uncertainty for solutions to inverse problems, Ocean Dynamics, 52, 71-78, 2002.

Yaremchuk, M. I., D. Nechaev, J. Schröter, and E. Fahrbach, A dynamically consistent analysis of circulation and transports in the southwestern Wedell Sea, Ann. Geophys., 16, 1024-1038, 1998.

Yaremchuk, M. I., N. L. Bindoff, J. Schröter, D. Nechaev, and S. R. Rintoul, On the zonal and meridional circulation and ocean transports between Tasmania and Antarctica, J. Geophys. Res., 106, 2815-2832, 2001.

M. Losch, Alfred-Wegener-Institut für Polar- und Meeresforschung Postfach 120161, 27515 Bremerhaven, Germany. (mlosch@awibremerhaven.de) 